\title{
On Interpolation by Iteration of Proportional Parts, without the Use of Differences
}

\author{
By A. C. Aтtкkn, Edinburgh University. \\ (Received 15th January, 1932. Read 5th February, 1932.)
}

§1. Introductory.

Linear interpolation between two values of a function $u_{a}$ and $u_{b}$ can be performed, as is well known, in either of two ways. If the divided difference $\left(u_{1}-u_{a}\right) /(b-a)$, which is usually denoted by $u(a, b)$ or $u(b, a)$, is provided, or its equivalent in tables at unit interval (the ordinary difference), we should generally prefer to use the formula

$$
u_{x}=u_{a}+(x-a) u(a, b),
$$

which is the linear case of Newton's fundamental formula for interpolation by divided differences. If differences are not given, but a machine is available, then the use of proportional parts in the form of the weighted average

$$
u_{x}=\left[(b-x) u_{a}+(x-a) u_{b}\right] /(b-a),
$$

the linear case of Lagrange's formula, is actually more convenient, since it involves no clearing of the product dials until the final result is read. In the usual case of data tabled at unit intervals the method of (1.2) is particularly simple, since the divisor $b-a$ is then unity or some small integer, and the division can be performed mentally. Even in the case of unequal intervals the process is convenient for machine, for the secondary dial which registers the turns, if it possesses " tens' transmission," will automatically add the multipliers $b-x$ and $x-a$ in (1.2), and will thus show the proper divisor $b-a$, which can then be used in division upon the productsum as registered by the product dials.

For example, a linear interpolate $u_{0.083}$ can be computed from $u_{0}$ and $u_{1}$ as

from $u_{0}$ and $u_{3}$ as

$$
\begin{aligned}
& u_{0.683}=0.317 u_{0}+0.683 u_{1} \\
& u_{0.683}=\left(2.317 u_{0}+0.683 u_{3}\right) / 3
\end{aligned}
$$

from $u_{1}$ and $u_{3}$ as$$
u_{0 \cdot 683}=\left(2.317 u_{1}-0.317 u_{3}\right) / 2 \text {, and so on. }
$$ 
It was without doubt the simplicity and ease of this operation by machine which led $\mathrm{Ch}$. Jordan to use the results as the basis of a new method of interpolation. He suggested (Metron, 7 (1928), 47-51 $)^{1}$ that instead of applying Bessel's or Everett's formula to a set of data $\ldots ., u_{-\frac{n}{2}}, u_{-\frac{1}{2}}, u_{\frac{1}{2}}, u_{\frac{n}{3}}, \ldots$ it is advantageous, if a machine is available, to take as the basis of further computation the results of linear interpolations for $u_{x}$ on the symmetrically disposed pairs of tabular values $u_{-\frac{1}{2}}$ and $u_{\frac{1}{3}} ; u_{-\frac{3}{3}}$ and $u_{\frac{3}{2}} ; u_{-\frac{5}{2}}$ and $u_{\frac{5}{3}}$, and so on, and to apply to these results a special formula of interpolation which uses even differences only.

The experience of computers has proved that Jordan's method is very rapid and effective, particularly when no tabular differences are provided. In the present paper we carry his idea still further by dispensing altogether with differencing and with the use of tables of interpolating coefficients, and we propose to carry out the whole process by iteration of the operation of proportional parts. Special attention is drawn to $\S 3$, in which the rapid and powerful operation of extrapolation by quadratic proportional parts is developed, and to the applications to double interpolation, and to inverse interpolation.

\section{§2. The Non-Symmetrical Case: Iteration of the Gregory-Newton} Type.

Consider first non-symmetrical interpolation. For vividness the data will be taken as equidistant, namely $u_{0}, u_{1}, u_{2}, u_{3}, \ldots$, but the demonstration will cover the more general case of $u_{a}, u_{b}, u_{c}, u_{d}, \ldots$ The routine is as follows. The first step is to interpolate for $u_{x}$ by proportional parts, as in $\S 1$, between $u_{0}$ and $u_{1}$; then between $u_{0}$ and $u_{2}$; then between $u_{0}$ and $u_{3}$, and so on. We shall write the results as

$$
u_{1}^{(1)}, u_{2}^{(1)}, u_{3}^{(1)}, \ldots,
$$

where the increase of suffix is to be noted, leaving the variable $x$ to be tacitly understood. Thus for example

$$
\begin{aligned}
& u_{1}^{(1)}(x)=u_{1}^{(1)}=(1-x) u_{0}+x u_{1}, \\
& u_{2}^{(1)}(x)=u_{2}^{(1)}=\left[(2-x) u_{0}+x u_{2}\right] / 2, \text { and so on. }
\end{aligned}
$$

1 For investigations of Jordan's formula, and of an allied formula suggested by the present writer where an odd number of tabular values are used, see articles in the Mathenatical Gazette, 16 (1932), 14-25. 
The second step is to treat the set (2.1) as data from which $u_{x}^{(1)}$ is to be estimated by the proportional part process, using the initial datum $u_{1}^{(1)}$ combined with each of the rest in turn. Let this sequence be written

$$
u_{2}^{(2)}, u_{3}^{(2)}, u_{4}^{(2)}, \ldots
$$

again with an increase in suffixes. For example,

$$
\begin{aligned}
& u_{2}^{(2)}=(2-x) u_{1}^{(1)}-(1-x) u_{2}^{(1)}, \\
& u_{3}^{(2)}=\left[(3-x) u_{1}^{(1)}-(1-x) u_{3}^{(1)}\right] / 2, \text { and so on. }
\end{aligned}
$$

The third step is similar; to interpolate linearly for $u_{v}^{(2)}$ from the set (2.2), using the initial $u_{2}^{(2)}$ combined with each of the rest in turn, leading to values $u_{3}^{(3)}, u_{4}^{(3)}$, and so on: and later steps follow the same routine.

We shall prove that $u_{s}^{(r)}$, as found by the above method, is identical with the value of $u_{x}$ as found by ordinary polynomial interpolation, with data $u_{0}, u_{1}, u_{2}, \ldots, u_{r-1}, u_{s}$. In particular the initial iterate of the $r^{\text {th }}$ sequence, $u_{r}^{(r)}$, gives the value of $u_{x}$ determined polynomially from $u_{0}, u_{1}, u_{2}, \ldots, u_{r}$.

To prove this, let $u_{a}, u_{b}, u_{c}, u_{d}$, for example, be a set of data. Denote the linear polynomial determined by $u_{a}$ and $u_{b}$ by $u_{\iota, b}$, the arbitrary variable $x$ being tacitly understood-very much in the way that a straight line through points $A$ and $B$ is denoted by $A B$; denote the quadratic determined by $u_{a}, u_{b}$ and $u_{c}$ by $u_{a, l, c}$; and so on. Then Newton's fundamental formula gives two identical results,

$$
\begin{aligned}
u_{a, b, c, b} & =u_{a, b, c}+(x-a)(x-b)(x-c) u(a, b, c, d), \\
& =u_{a, b, d}+(x-a)(x-b)(x-d) u(a, b, c, d),
\end{aligned}
$$

where $u(a, b, c, d)$ denotes the divided difference of the third order arising from $u_{\alpha}, u_{b}, u_{c}, u_{d}$. Eliminating the last terms in (2.3) and (2.4) by multiplying by $d-x$ and $x-c$ respectively and adding, we have

$$
u_{a, b, c, d}=\left[(d-x) u_{a, b, c}+(x-c) u_{a, b, d}\right] /(d-c) .
$$

But this is merely the use of proportional parts, as if we were interpolating linearly for $u_{a, b, v}$ from $u_{a, b, c}$ and $u_{a, b, d}$, regarded as functions of $x=c$ and $x=d$. The algorithm of iteration consists now in the repeated use of the identity (2.5). For example what was denoted by $u_{1}^{(1)}$ is seen to be the same as $u_{0,1}$, while $u_{2}^{(1)}$ is the same as $u_{0,2}$; so 
that, in virtue of $(2.5), u_{2}^{(2)}$ is the same as $u_{0,1,2}$; and similarly for every other entry. It follows at once that all entries in the sequence $u^{(r)}$ are interpolates for $u_{i}$ corresponding to some polynomial of the $r^{\text {th }}$ degree, and so are correct to $r^{\text {th }}$ differences.

This last remark has a practical bearing, for since the iterated sequences are closer and closer approximations to the desired value, their earlier and more important significant digits tend to become the same throughout the sequence, so that only the later different ones need to be copied down and used in forming the weighted averages at subsequent stages.

In practice one begins this process early by dropping figures common to all the original u's: e.g. most of the 5's in the table below.

Even though the present non-symmetrical method of iteration will be passed over in nearly all practical cases, except that of inverse interpolation, considered in $\$ 7$, in favour of the quadratic extrapolative process of the next sections, which is from two to three times as rapid, it may be interesting to see the features just mentioned coming out in a numerical example. We shall take the first six values of the Digamma function, $d \log _{e}(x !) / d x$, retaining eight places of the twelve-place values given in the B. A. Tables (London, 1931), p. 42 , for $x=\cdot 00, \cdot 01, \ldots, \cdot 05$, and we shall interpolate for $x=0268327$. The values are all negative, but we shall work with the positive sign, prefixing a minus to the result. The following is the most convenient arrangement:

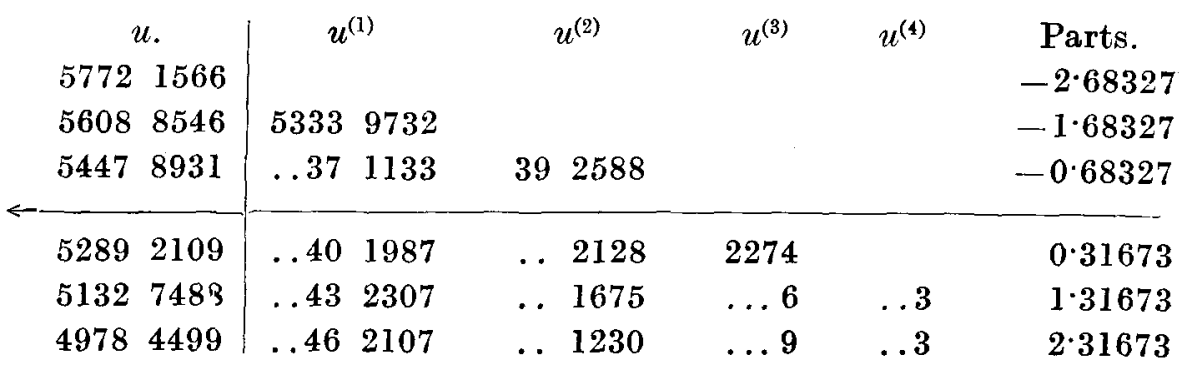

Hence we have $\quad u_{x}=-0.53392273$.

The appropriate multipliers in the operations of proportional parts are shown in the column on the right. If we imagine the 
values of the $u^{\prime}$ 's and the $u^{(r)}$ 's as being ordinates, all entries in the same row having the same abscissa or value of $x$, then these multipliers are seen to be the distances of the ordinates corresponding to suffixes $0,1,2, \ldots$ from the desired ordinate with suffix $x$, and, further, they are used in pairs in ordinary cross-multiplication upon entries in their rows.

For example, the first entry in the column $u^{(1)}$ is given by

$$
2 \cdot 68327 \times 56088546-1.68327 \times 57721566,
$$

the secondary dial registering 1.00000. The last entry in the same column is given by

$$
(2 \cdot 68327 \times 49784499+2 \cdot 31673 \times 57721566) / 5,
$$

the secondary dial recording the divisor 5.00000. Again, the first entry in the column $u^{(2)}$ is given by

$$
1 \cdot 68327 \times 371133-0.68327 \times 339732,
$$

while the last entry in the same column is given by

$$
(1 \cdot 68327 \times 462107+2 \cdot 31673 \times 339732) / 4 \text {, and so on. }
$$

The entry 2274 in the column $u^{(3)}$ is given by $0.68327 \times 2128+$ $0.31673 \times 2588$. As fewer and fewer digits are retained it becomes pointless, of course, to retain all the digits in the multipliers. Visual help is given if a horizontal datum line, with arrowhead as indicated, be drawn across the table in a position which will approximately represent the desired ordinate of interpolation, for then the sign and magnitude of the distance-ratios which are used as multipliers are made manifest. Finally the fact that the decimal parts of all multipliers involve two numbers only, namely 0.68327 and its complement 0.31673 , renders the process uniform and congenial.

We pass now to the symmetrical case, which possesses all of the above advantages in an enhanced form.

\section{§3. Quadratic Extrapolation for an Even Number of Data.}

We consider this time a set of $2 n$ symmetrically disposed data, not necessarily equidistant, but such as $u_{-c}, u_{-b}, u_{-a}, u_{a}, u_{b}, u_{c}$. These determine a polynomial of degree $2 n-1$, which we shall denote by $u_{ \pm a, \pm b, \pm c}$, the variable $x$ being again tacitly understood. 
We shall obtain this polynomial by iterating upon an initial sequence $u_{ \pm ! \prime}, u_{ \pm b}, u_{ \pm c}$, that is, upon the symmetrical linear interpolates which Jordan used. Writing down the value of the linear polynomial $u_{ \pm a}$, namely

$$
u_{ \pm a}=\left[(a+x) u_{a}+(a-x) u_{-a}\right]_{/} / 2 a,
$$

we observe that it is unchanged by writing $-a$ for $a$. Hence it is an even function of its argument $a$, and so might be denoted by $f\left(x ; a^{2}\right)$. We also note that if in it we put $a=x$, we obtain $u_{x}$. Hence $u_{x}$ is the same as $f\left(x ; x^{2}\right)$. It follows at once that we can interpolate for $u_{x}$ from the values $f\left(x ; a^{2}\right)$, or $u_{ \pm a}$, by the formula 2.5 of $\S 2$, but with squared variables, obtaining the fundamental formula of iteration,

$$
u_{ \pm a, \pm b, \pm c}=\left[\left(c^{2}-x^{2}\right) u_{ \pm a, \pm b}+\left(x^{2}-b^{2}\right) u_{ \pm a, t_{c}}\right] /\left(c^{2}-b^{2}\right),
$$

which can evidently be extended to any even number of symmetrically placed data.

By the repeated use of (3.2) we develop the method of iterated quadratic extrapolation as follows. The first step is to compute the Jordan interpolates $u_{ \pm a}, u_{ \pm b}, u_{ \pm c}, \ldots$ by ordinary linear proportional parts, as in (3.1). The next step is to apply formula (3.2) to $u_{ \pm a}$ and $u_{+b}$, obtaining $u_{ \pm a, \pm b}$; then to $u_{ \pm a}$ and $u_{ \pm c}$, obtaining $u_{ \pm a, \pm c}$, and so on. The third step is to apply (3.2) again to $u_{ \pm a, \pm b}$ and $u_{ \pm a, \pm c}$, obtaining $u_{ \pm a, \pm b, \pm c}$, and so on. At the second step we obtain interpolates to third differences, at the third step to fifth differences, and so on. The various sequences can be arranged in columns as in $\S 2$, though we shall see that the computation is best carried out by building downwards in a triangular fashion, annexing rows as required.

A valuable feature of the method is that the quadratic multiplier, for instance $c^{2}-x^{2}$ in (3.2), is a product of $c+x$ and $c-x$, which have already been used as linear multipliers in the first Jordan step. This is peculiarly useful in the case of equidistant data at unit intervals; for then we have $a=\frac{1}{2}, b=\frac{3}{2}, c=\frac{5}{2}$, and so on, so that the quadratic multipliers to be used in the iterations are

$$
\frac{1}{4}-x^{2}, \frac{1}{4}-x^{2}, \frac{2 z}{4}-x^{2}, \frac{+9}{4}-x^{2}, \ldots,
$$

that is, $\theta, 2+\theta, 6+\theta, 12+\theta, 20+\theta, 30+\theta$, taking them as far as would be required for interpolation to eleventh differences, where $\theta=\left(\frac{1}{2}+x\right)\left(\frac{1}{2}-x\right)$. It is advisable therefore, before ever carrying 
out the first central Jordan interpolation, to compute and note down for use-a matter of a few moments-the product of the linear multipliers which are being used. The value of $\theta$ thus obtained recurs throughout the whole iteration, the integers $2,6,12,20, \ldots$. and their mutual differences being divisors.

By way of illustration of these various points we shall work through an example given in the Introduction, pp. ix and $\mathrm{x}$, to the B.A. Tables of 1931, cited earlier. The problem is to interpolate for $S i(x)$, when $x=22 \cdot 12742983$, from entries given (ibid.) in Table $V I I I$, p. 38. The intervals are of 0.2 , the two central values, for example, being

$\begin{array}{cc}x & \text { Si }(x) . \\ 22 \cdot 0 & 1 \cdot 6160837366 \\ 22 \cdot 2 & 1 \cdot 6151035866 .\end{array}$

The central linear multipliers are therefore $0.63714915,0.36285085$, and their product, the quadratic multiplier $\theta$, is 0.23119011065 . We read from the table the values of the function at widening ranges about the central interval, set them up on the machine with the proper linear multipliers, i.e. the above ones, with a unit added to each each time, divide the results by $1,3,5,7, \ldots$, and enter the Jordan sequence $u^{(1)}$ in the computing sheet. Actually all that is written down on the sheet appears below:

\begin{tabular}{|c|c|c|c|c|c|}
\hline$u^{(1)}$ & $u^{(3)}$ & $u^{(5)}$ & $u^{(7)}$ & $u^{(0)}$ & Parts \\
\hline $1 \cdot 6154592348 \cdot 6$ & & & & & 0.23119011065 \\
\hline $136866907 \cdot 4$ & $156641322 \cdot 0$ & & & & $2 \cdot 23 \ldots$ \\
\hline $102107315^{\circ} 4$ & $614685 \cdot 4$ & $656179 \cdot 8$ & & & $6 \cdot 23 \ldots$ \\
\hline $051669644 \cdot 1$ & $575241 \cdot 2$ & $6065 \cdot 9$ & $6298 \cdot 1$ & & $12 \cdot 23$. \\
\hline $59875 \quad 22727 \cdot 9$ & $523590 \cdot 8$ & $5915 \cdot 4$ & $7 \cdot 5$ & $9 \cdot 0$ & $20 \cdot 23 \ldots$ \\
\hline
\end{tabular}

Thus we have $S i(22 \cdot 12742983)=1 \cdot 6156656299$.

It is expedient to do the computation in the following order. The first entry in the column $u^{(3)}$ is given by

$$
(2 \cdot 23119 \ldots \times 545923486-0.23119 \ldots \times 368669074) / 2,
$$

and the second entry by

$$
(6.23119 \ldots \times 545923486-0.23119 \ldots \times 021073154) / 6,
$$


the quadratic multipliers $\theta, 2+\theta, 6+\theta$, being used in crossmultiplication upon the entries in their rows, exactly as were the linear multipliers of $\S 2$. Since the results obtained at this stage are by no means equal or nearly equal, we must proceed to higher differences, and so we compute a third entry in column $u^{(3)}$ by

$$
(12.23119 \ldots \times 1545923486-0.23119 \ldots \times 0516696441) / 12 .
$$

From the three values in column $u^{(3)}$ we now calculate the two first values in column $u^{(5)}$. Though much closer to equality, they are not yet sufficiently near for our purpose. Hence we compute a fourth entry in the column $u^{(3)}$, and by means of it a third entry in the column $u^{(5)}$. The three entries in column $u^{(5)}$ now give two (to seventh differences) in the column $u^{(7)}$, and these are less than a unit apart. Hence we proceed with assurance to the single entry in column $u^{(9)}$, the extrapolating ratio $20:-12$, or $5:-3$, being sufficient. Thus we build downwards in triangular fashion by annexing rows, but only adding the apex at the very last step.

We adopt the above procedure, which is systematic and easy, in order not to extend the columns unnecessarily, and yet to check the final result. In the above example it has been necessary, for accuracy to ten decimals, to go as far as the equivalent of ninth differences. In common practice it will rarely be necessary to go beyond fifth differences, and indeed, had the tables of $S i(x)$ been entered at interval 0.1 instead of 0.2 , a fair proportion of our work would have been saved.

It may be noted that the decimal part of all the quadratic multipliers is the same, namely $0.23119 \ldots$; that all the divisors are integers, shown on the secondary dial; that fewer and fewer digits are copied down, or used in the multipliers, as the iteration proceeds, and with a much better convergence than in the linear case of $\S 2$; that whereas tables of interpolating coefficients have to be restricted for reasons of space to three-digit values of $x$ between 0 and 1 , in the present method $x$ is unrestricted both in range and in number of digits, so that (i) the method can be employed close to the end of a table, (ii) there is no necessity for calculating adjacent values and for sub-interpolating upon them; finally, the convergence to the desired result is presented to the eye of the computer throughout. These several features seem to be advantageous in interpolation with machine aid. 


\section{§4. Procedure for an Odd Number of Symmetrical Data.}

The method described in $\S 3$ has a certain analogy with the Newton-Bessel (or with the ordinary Everett) formula, and gives of course equivalent results. There is a similar iterative process, analogous to the Newton-Stirling (or to the second Everett) formula, which is applicable to an odd number of symmetrically placed data, such as $u_{-c}, u_{-b}, u_{-a}, u_{0}, u_{a}, u_{b}, u_{c}$. We write

$$
u_{x}=u_{0}+x v_{v},
$$

so that $v_{x}$ is the divided difference $u(0, x)$, and we iterate for $v_{x}$ instead of $u_{x}$, taking as data $v_{a}$, that is, $u(0, a)$, and $v_{-a}, v_{b}$ and $v_{-b}$, and so on, and forming from them a Jordan sequence $v_{ \pm a}, v_{ \pm b}, v_{ \pm c}, \ldots$, to which we apply the formula (3.2) as before. When the iteration has been carried far enough we complete the calculation by means of (4.1).

The initial sequence $v_{ \pm a}, v_{ \pm b}, v_{ \pm_{c}}, \ldots$ can be computed in either of two ways. One is to evaluate the divided differences $u(0, a)$, $u(0,-a)$, and so on, which in the case of equidistant data at unit interval means subtracting adjacent data from the central one and dividing the result by $1,2,3, \ldots$, and to interpolate on these by proportional parts as if for $u(0, x)$, so that we have

$$
v_{ \pm a}=[(a+x) u(0, a)+(a-x) u(0,-a)] / 2 a .
$$

This is of course quite simple and rapid, but the writer personally prefers to do the computation from the tabular data in one step on the machine by the equivalent formula

$$
v_{ \pm a}=\left[(a+x) u_{a}-2 x u_{0}-(a-x) u_{-a}\right] / 2 a^{2},
$$

the secondary dial showing a zero total. For seven equidistant data this requires a division by 18 , and for nine data one by 32 , but these can be done rapidly on the machine, if not mentally. It will probably be better to compute the two outer terms in the bracket of (4.3) first, and to subtract the $2 x u_{0}$ last; indeed the $2 x u_{0}$, which occurs in each $v_{ \pm \text {s, }}$ might be computed once and for all in readiness.

We now proceed to operate upon the $v^{(1)}$ 's just as we did upon the $u^{(1)}$ 's in $\S 3$, but we must note that in the equidistant case the values of $a, b, c, \ldots$ are now $1,2,3, \ldots$ instead of $\frac{1}{2}, \frac{3}{2}, \frac{5}{2}, \ldots$ as formerly, so that $\theta$, the first quadratic multiplier, is $1-x^{2}$, and the rest are

$$
3+\theta, 8+\theta, 15+\theta, 24+\theta, \text { and so on. }
$$


It happens that $\theta$ is again the product of the linear multipliers used in (4.2) or (4.3), a fact that may be turned to the same advantage as before.

By way of illustration we shall work again through the example of $\S 3$, taking $S i(22 \cdot 2)$ for central datum and reading the data up the table in reverse order, so that $x=0 \cdot 36285085$. We use (4.3) directly on the machine, so that all that appears in the computing scheme is as follows:

Linear multipliers for $v^{(1)}, 1 \cdot 36285085 ; 0.63714915 ; 0 \cdot 72570170$. Product $\theta=0 \cdot 8683392607$.

\begin{tabular}{|c|c|c|c|c|c|}
\hline & $v^{(1)}$ & $v^{(3)}$ & $v^{(5)}$ & $v^{(7)}$ & Parts. \\
\hline 153 & $45306 \cdot 1$ & & & & $0.86833 \quad 92607$ \\
\hline 48 & $51679 \cdot 3$ & $5488184^{\prime} 6$ & & & $3 \cdot 868$ etc. \\
\hline 40 & $46254 \cdot 6$ & $6308 \cdot 3$ & $9636 \cdot 2$ & & $8 \cdot 868 \ldots$ \\
\hline 29 & $54337 \cdot 8$ & $3717 \cdot 5$ & $24 \cdot 6$ & $50 \cdot 9$ & $15 \cdot 868 \ldots$ \\
\hline
\end{tabular}

Hence $u_{x}=1.6151035866+0.36285085 \times 0.00154896509$

$$
=1 \cdot 6156656299 \text {. }
$$

For example, the three central values in the table of $S i(x)$ being

$$
\begin{aligned}
& u_{1}=1 \cdot 6160837366 \\
& u_{0}=1 \cdot 6151035866 \\
& u_{-1}=1 \cdot 6123832456,
\end{aligned}
$$

we calculate the first entry in the column $v^{(1)}$ by means of $\frac{1}{2}(1.36285085 \times 60837366-0.63714915 \times 23832456-0.72570170 \times 51035866)$, the next entry by

$$
\frac{1}{8}\left(2 \cdot 36285 \ldots \times u_{2}-1 \cdot 63714 \ldots \times u_{-2}-0.72570 \ldots \times u_{0}\right)
$$

and so on by (4.3), reading the values of $u_{a}$ from the tables. Quadratic extrapolation is then carried out upon the values $v^{(1)}$ in exactly the same way as in $\S 3$, with multipliers $\theta, 3+\theta, 8+\theta, 15+\theta$.

We have included this section for completeness of exposition, but in many cases it may be thought easier not to interpolate in this manner from $2 n-1$ data, but to annex a further value and use the method of $\S 3$ for $2 n$ data, which is more straightforward. On the 
other hand, the computation of the values $v^{(1)}$ on the machine by (4.3) is nearly as easy, after a little practice, as the computation of the values $u^{(1)}$ in $\S 3$, and since the $v^{(1)}$ 's are of the order of first differences, they have fewer digits than the $u^{(1)}$ 's, and consequently require fewer digits in the quadratic multipliers. Our own experience is that there is not much difference in speed between the two methods.

The quadratic multipliers $\theta, 3+\theta, 8+\theta, \ldots$, which appear in the present process of "mid-point" iteration occur naturally also in the problem of interpolating from a symmetrical set of equidistant data which lacks its middle entry, such as $u_{-3}, u_{-2}, u_{-1}, u_{1}, u_{2}, u_{3}$. As an example we shall extract from the tables of the Digamma function mentioned in $\S 2$ the first seven values, to ten digits. We shall delete the fourth value and try to recover it from the remaining six by the present method. Here it happens that $\theta=1$, so that the quadratic multipliers are simply the square integers $1,4,9$. The ratios used are therefore as the squares of the ranges of interpolation selected, so that our first quadratic approximation coincides with a process which has been called by L. F. Richardson " $h^{2}$-extrapolation." [See, for example, The Deferred Approach to the Limit, by L. F. Richardson and J. A. Gaunt, Phil. Trans. 226 A (1927), 299-361.] Our linear interpolates $u^{(1)}$ are here simply the averages of symmetrically placed data. The work appears as follows:

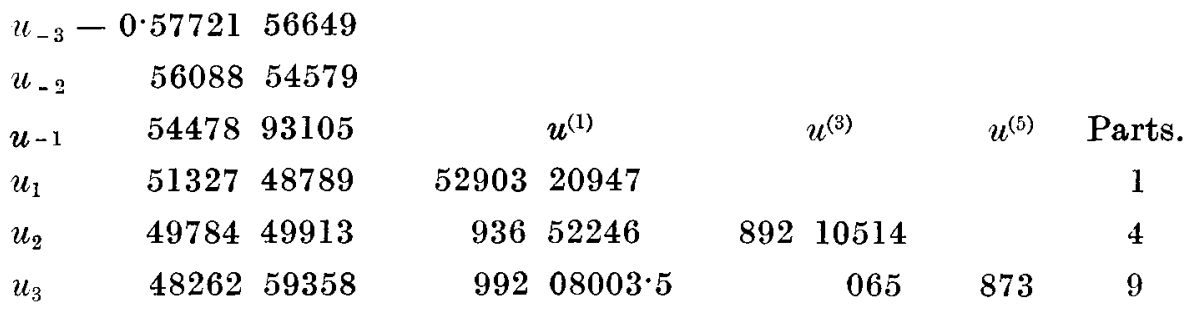

The required value is thus -0.5289210873 , which agrees to the last digit with the tabular value. A procedure of equal simplicity and convenience for " interpolating to halves" from any even number of symmetrically placed equidistant (or even not equidistant) data can easily be arranged.

\section{\$5. Quadratic Extrapolation at the End of a Table.}

Before we leave the subject of interpolation by these methods for functions of one variable, we shall give a numerical example of interpolation close to the end of a table. We shall interpolate 
upon the six values of the Digamma function given in $\S 2$, for $x=0.0031673$. The central linear multipliers are therefore $2 \cdot 68327$, $-1 \cdot 68327$, and their product $\theta$ is $-4 \cdot 5166679$. The $u^{(1)}$ 's are calculated as before, from the two central values outward, and the appearance of the computing scheme differs from the earlier examples only in the fact that $\theta$ is negative and numerically exceeds unity.

\begin{tabular}{|c|c|c|c|c|}
\hline$u^{(1)}$ & & $u^{(3)}$ & $u^{(5)}$ & Parts. \\
\hline $5714 \quad 9980 \cdot 9$ & & & & $-4 \cdot 5166679$ \\
\hline $172908 \cdot 7$ & 20 & $1759 \cdot 6$ & & -2.5166679 \\
\hline $218784 \cdot 6$ & & $74 \cdot 8$ & $69 \cdot 1$ & $1 \cdot 4833321$ \\
\hline
\end{tabular}

Hence we have

$$
u_{s}=-0.57201769 \text {. }
$$

It appears therefore that quadratic extrapolation works just as well, except, in general, for a somewhat less rapid convergence, when the required interpolate does not lie within the central "panel", but at some distance away. This does away with the need for any modification of formulae or procedure at the ends of a table.

\section{§6. Interpolation for Functions of Two Variables.}

The method of quadratic extrapolation can be applied, with proportionate, but not disproportionate, increase of work, to the problem of interpolation upon a function of two variables $u_{x, y}$, the basis of iteration being a preliminary calculation of interpolates linear in $x$ and also in $y$, that is, bilinear in both together, by a double use of Jordan's process. It is assumed therefore that $u_{x, y}$ can be approximately represented, not by a double polynomial in $x$ and $y$ of degree $n$ in both together, and so given by $(n+1)(n+2) / 2$ values disposed in triangular shape on a table of double entry, but by an $m$-ic in $x, n$-ic in $y$ type of polynomial. (Cf. Steffensen's Interpolation, p. 203.)

In the simplest case the values of $u_{x . y}$ will appear at points in a rectangular lattice, in rows and columns. The rows and columns about the central rectangle within which the desired interpolate lies will be denoted by row $_{\frac{1}{2}}$, row ${ }_{-\frac{1}{2}}$ and $\operatorname{col}_{\frac{1}{2}}$, col ${ }_{-\frac{1}{2}}$; those further out on either side by row row $_{-\frac{3}{2}}$ and $\mathrm{col}_{3}, \mathrm{col}_{-\frac{3}{3}}$; and so on. The first step is to interpolate linearly for $x$ between row $_{\frac{1}{2}}$ and row for $_{-\frac{1}{2}}$ a few values of $y$ on either side of the central value, writing down the results in a row; then similarly between row $_{\frac{3}{2}}$ and row ${ }_{-\frac{3}{2}}$, writing 
down the results in a row below the other; then between row row ${ }_{-\frac{3}{2}}$, and so on. On the rows so obtained linear interpolation is then performed for $y$ between $\mathrm{col}_{\frac{1}{2}}$ and $\mathrm{col}_{-\frac{1}{2}} \mathrm{col}_{\frac{3}{2}}$ and $\mathrm{col}_{-\frac{3}{2}}$; and so on. Thus the original $2 r$-by- $2 s$ lattice of data has been replaced by an $r$-by-s lattice of bilinear interpolates for the desired value of $x$ and $y$, the procedure being easy to understand and carry out.

The values in each column are now subjected to quadratic extrapolation, in the manner of $\S 3$, for the same value of $x$ and consequently with the same values of the quadratic multipliers $\theta, 2+\theta, 6+\theta$, etc., which are used over and over again. When the convergence in each column is seen to be sufficient the resulting row of values is subjected to quadratic extrapolation for $y$ in the same way. All that is required, for data equidistant in $x$ and in $y$, in the whole process is therefore the two complementary linear multipliers for $x$ and $y$, and their respective products $\theta$ and $\phi$, the quadratic multipliers.

As an example we shall take the 36 entries $u_{x, y}$ given on $p .12$ of Tracts for Computers, No. III (Cambridge, 1920). The illustrative example on page 11 of that work takes $x=\frac{1}{2}, y=\frac{1}{2}$, using what is really a double Everett formula. We shall choose $x=0.479312$, $y=0.513621$. The 18 values obtained by central linear interpolation for $x$ are then computed, by setting up from the table on to the machine, as:

$\begin{array}{rrrrrr}8981904 \cdot 2 & 925467 \cdot 6 & 866954 \cdot 9 & 806361 \cdot 1 & 743687 \cdot 4 & 678935 \cdot 9 \\ 923005 \cdot 5 & 864220 \cdot 6 & 803406 \cdot 6 & 740568 \cdot 7 & 675717 \cdot 5 & 608866 \cdot 2 \\ 805087 \cdot 8 & 741797 \cdot 3 & 676590 \cdot 9 & 609493 \cdot 4 & 540534 \cdot 3 & 469746 \cdot 5 .\end{array}$

From these by central linear interpolation for $y$ we compute the nine bilinear interpolates; and the rest of the work is as below.

\begin{tabular}{cccc|l}
88 & $35832 \cdot 7$ & $33752 \cdot 2$ & $29594 \cdot 7$ & $\theta=0 \cdot 2495720$. \\
87 & $71131 \cdot 7$ & $69113 \cdot 2$ & $65080 \cdot 1$ & $2+\theta$ \\
86 & $42128 \cdot 2$ & $40252 \cdot 0$ & $36503 \cdot 6$ & $6+\theta$ \\
& & & & \\
88 & $43906 \cdot 5$ & $41818 \cdot 2$ & $37645 \cdot 3$ & $2+\theta$ \\
& $43889 \cdot 9$ & $41800 \cdot 9$ & $37626 \cdot 4$ & $6+\theta$ \\
$43915 \cdot 8$ & $41827 \cdot 9$ & $37655 \cdot 9$ & \\
$\phi$ & $2+\phi$ & $6+\phi$ & $\phi=0 \cdot 2498145$. \\
\multicolumn{4}{c}{$44176 \cdot 6$} & $44176 \cdot 4$
\end{tabular}

$44176 \cdot 7$. 
Hence $u_{x_{, y}}=0.8844177$, which is probably in defect of the true value by two units in the last place, since the value found by Pearson for $u_{\frac{1}{2}, \frac{1}{2}}$, as computed by the double Everett formula, is in defect by this amount.

Most of the time consumed in the above computation was taken up in obtaining the nine bilinear interpolations, for which 27 operations, each involving the addition of two products, with possible division by 3 or 5 , were required. Once the bilinear basis is obtained the double quadratic extrapolation is most rapidly set going. Hence it becomes a question how to accelerate the calculation of the bilinear interpolates. The writer found the following to be an effective method. From the appropriate linear multipliers for $x$, as in the present case $2+\xi, 1+\xi, \xi, x, 1+x, 2+x$, where $\xi=1-x$, and those for $y$, namely $2+\eta, 1+\eta, \eta, y, 1+y, 2+y$, where $\eta=1-y$, construct a multiplication table showing the products of these. It is seen at once that all of the entries in any row or column of this table of bilinear multipliers are (apart from a change of sign in passing from $\xi$ to $x$, and from $\eta$ to $y$ ), in arithmetical progression, and so can be computed from the first entry in that row or column by pure subtraction, that is, turning the handle of the machine and writing down the answer. For example in the present case the table of bilinear multipliers for sixteen central entries is

$$
\begin{array}{rcccc} 
& 1+\eta & \eta & y & 1+y \\
1+\xi & 2 \cdot 2603187 & 0.7396307 & 0.7810573 & 2 \cdot 3017453 \\
\xi & 0.7739397 & 0.2532517 & 0.2674363 & 0.7881243 \\
x & 0.7124393 & 0.2331273 & 0.2461847 & 0.7254967 \\
1+x & 2 \cdot 1988183 & 0.7195063 & 0.7598057 & 2 \cdot 2391177
\end{array}
$$

which are readily computed from the leading coefficient $2 \cdot 2603187$ alone, by subtraction, retaining an extra digit for accuracy, reading " nines-complements" when we come to negative numbers, and filling in the first row and column first. Indeed we can work spirally round the table, if we prefer, clearing nothing on the product dials, adding or subtracting as required, and finishing up at the centre. We use these bilinear multipliers upon sets of four entries in the table of data which form rectangles centrosymmetric with respect to the central rectangle, applying to each datum the corresponding multiplier in the 
table.: For example the eight central values in the two central rows of the table of $u_{x, y}$, namely

$\begin{array}{llll}\cdot 8793305 & \cdot 8728127 & \cdot 8660736 & \cdot 8591144 \\ \cdot 9069039 & \cdot 9017767 & \cdot 8964557 & \cdot 8909399,\end{array}$

give rise to the two bilinear interpolates,

and

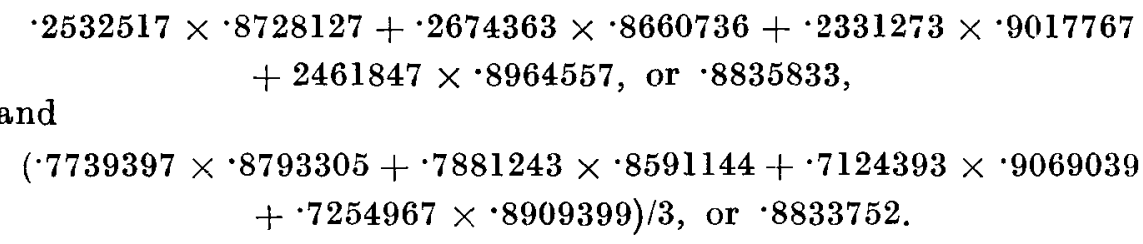

For values of $x$ and $y$ given to a few decimals only such a table of bilinear multipliers can be prepared in a few minutes.

It is evident that we can similarly extend to two variables the " mid-point" quadratic extrapolation process of $\S 4$, and also that we can combine the methods, using one for $x$, say, on an even number of rows, the other for $y$ on an odd number of columns. It is probable that the "mid-point" method, whether used for both variables or only for one, will not prove to be as useful (unless interpolation as far as second differences is sufficiently accurate for requirements) or as easy to apply as the double use of the method of $\S 3$ which has just been exemplified, and we shall not delay to examine it in detail.

One marked advantage of the method of double quadratic iterative extrapolation has been observed in the illustrative example, namely that the full and exact values of $x$ and $y$ can be used right from the beginning, without any need for taking adjacent values to three digits and sub-interpolating on the four or more results. Also, as in the case of one variable, the ordinate of interpolation is not restricted to lie within the central rectangle, but may possibly be close to the edge of a table, the only effect being a somewhat slower convergence of the iterates.

A final remark may be made. The interpolation upon 36 entries which we have used for illustration is equivalent to the assumption that the function $u_{x, y}$ can be represented over the range in question, to the desired order of accuracy, by a double-quintic polynomial in $x$ and $y$. Had the interval of tabulation been reduced to one quarter, let us say, of what it is in the table, a double cubic would probably have been adequate, and this can be interpolated for on 16 points 
with a very considerable saving of time as compared with the 36 point case, for the basis then consists of four bilinear interpolates only, and quadratic extrapolation on these involves only three operations.

\section{§7. Application of Linear Iteration to Inverse Interpolation.}

There is one case where quadratic extrapolation cannot be applied because symmetry is never present, but where the linear iteration of $\S 2$ can be used very effectively. This is in inverse interpolation. Here we have several values of a function $y$ corresponding to several adjacent, but not necessarily equidistant, values of $x$, and we wish to find what value of $x$ in the range corresponds to some prescribed value of $y$. Under the usual assumptions of onevaluedness of $x$ in the range, and of expansion as a sufficiently convergent Taylor series in $y$, we can interchange the variables and proceed to interpolate by iteration of linear proportional parts as in $\S 2$, the only difference now being that whereas before we performed the divisions by small integers mentally, we now perform divisions by numbers of several digits on the machine. The procedure will be clear from an example, p. 61 of Whittaker and Robinson's Calculus of Observations, the finding by inverse interpolation of the positive root of the equation

$$
y=z^{7}+28 z^{4}-480=0,
$$

from values of $y$ given by $z=1 \cdot 90,1 \cdot 91,1 \cdot 92,1 \cdot 93,1 \cdot 94$. In this case, since the desired value of $z$ corresponds to $y=0$, the given values of $y$ are the "distances of the ordinates" which were used as ratios in $\$ 2$, and so are themselves the linear multipliers. The computation is shown below.

\begin{tabular}{rrcrrr}
\multicolumn{1}{c}{$y$} & \multicolumn{1}{c}{$z^{(1)}$} & $z^{(2)}$ & $z^{(3)}$ & $z^{(4)}$ \\
$-25 \cdot 7140261$ & $1 \cdot 90$ & & & & \\
$-14 \cdot 6254167$ & 1 & 23189586 & & & \\
$-3 \cdot 3074639$ & 2 & 2952228 & 2882864 & & \\
\hline $8 \cdot 2439435$ & 3 & 2716929 & 87312 & 84138 & \\
$20 \cdot 0329830$ & 4 & 2483678 & 91702 & 17 & 53
\end{tabular}

Hence the value of $z$ to 9 places of decimals is 1.922884153 , which is in agreement with the result given to 10 places in Whittaker and Robinson. 
The process hardly calls for description. The values of $y$ are used in cross-multiplication ${ }^{1}$ on the entries of $z$ in their rows. The secondary dial records the differences, or sums, of these multipliers. These are set up on the divisor dials, and division is performed on the result shown on the product dials. The process demands no calculation of differences, no formulae of interpolation by differences and no awkward successive approximations, only the single operation of cross-multiplication and division just described. It is highly suited to machine work.

\section{§8. Concluding Observations.}

A few remarks of a desultory nature, which do not bear so directly on the work of the earlier sections, may be appended.

(1) The process of cross-multiplication which was used in the linear iterations of $\S 2$ can be regarded as a way of arranging computation by Lagrange's formula. Consider for example four data, $u_{a}, u_{l}, u_{c}, u_{d}$. The linear multipliers are, with change of sign, $x-a, x-b, x-c, x-d$. Inspection of the mode of cross-multiplication shows that any datum, for example $u_{b}$, is multiplied ultimately by all the linear multipliers except the one in its own row, with corresponding divisors. Hence the contribution it makes to the desired interpolate is

$$
u_{b}(x-a)(x-c)(x-d) /(b-a)(b-c)(b-d),
$$

and the sum of these for $u_{a}, u_{b}, u_{c}, u_{d}$ is just Lagrange's formula.

(2) The following alternative justification of the linear iteration of $\$ 2$ may be of interest:

Consider for example the cubic polynomial $u_{c c}$, or $u_{a, b, c, d}$, determined by $u_{a}, u_{b}, u_{c}, u_{d}$. By Newton's formula, we have

$$
\begin{aligned}
& u_{a, b, c}=u_{a, b}+(x-a)(x-b) u(a, b, c), \\
& u_{a, b, d}=u_{a, b}+(x-a)(x-b) u(a, b, d) ;
\end{aligned}
$$

but we also have exactly

$$
u_{a, b, c, d}=u_{a, b}+(x-a)(x-b) u(a, b, x) .
$$

Now evidently $u(a, b, x)$, being the divided difference of the second order of a cubic, is linear in $x$. Hence it may be found

1 It is advisable to do the work well to the left of the machine, in order to leave room for the division process, the divisor having several digits. 
exactly by proportional parts from $u(a, b, c)$ and $u(a, b, d)$, considered as functions of $x=c$ and $x=d$. This gives at once, by (i) and (ii), the result

$$
u_{a, b, c, d}=\left[(d-x) u_{a, b, c}+(x-c) u_{a, b, d}\right] /(d-c),
$$

that is, the fundamental identity (2.5).

(3) The linear iteration by proportional parts of $\S 2$ has also a geometrical meaning, and leads to a simple construction for geometrical interpolation, different from Newton's mixed arithmetical and geometrical construction. [See D. C. Fraser's Newton's Interpolation Formulas (London, C. and E. Layton), p. 83, or J.I.A, 58 (1927), 83.] For example, let $A P, B Q, C R, D S$ be given ordinates, not necessarily equidistant, of the curve of a cubic polynomial, and let it be required to find where the curve cuts some prescribed ordinate $X T$.

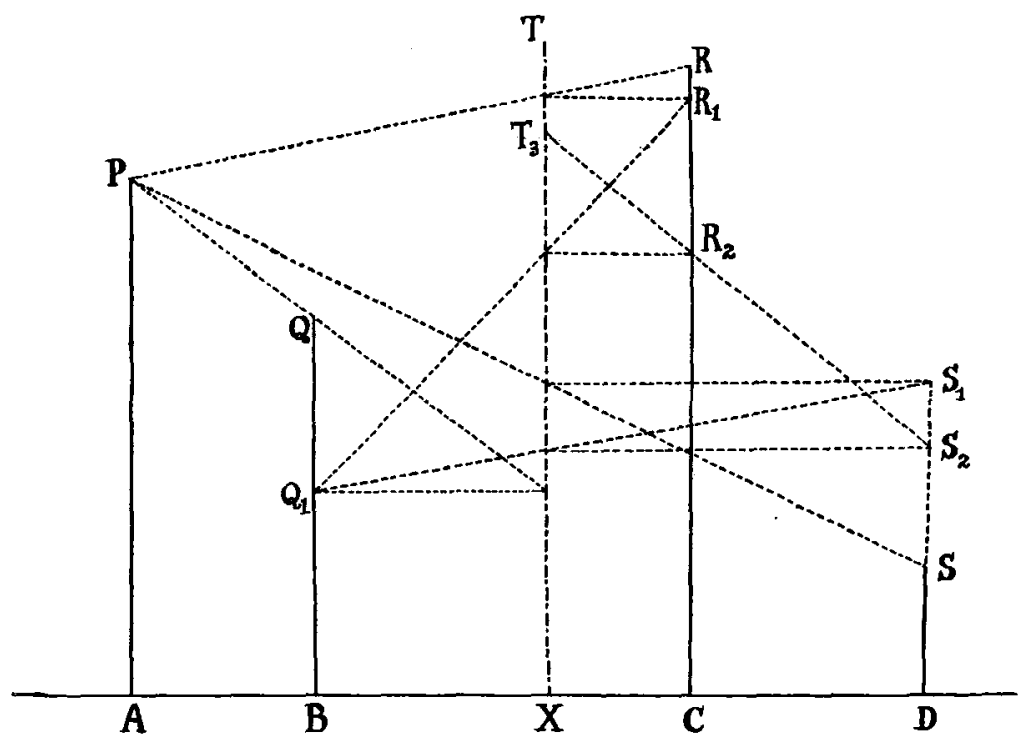

Translating the argument of $\S 2$ into geometrical terms, we carry out the following constructions:

I. Join $P Q$ and let the straight line $P Q$ cut $X T$. From the point of intersection draw a perpendicular to meet $B Q$ in $Q_{1}$.

Join $P R$ to cut $X T$ similarly, and from the point of intersection let a perpendicular be drawn to meet $C R$ in $R_{1}$, 
Join $P S$ and in exactly the same way determine a point $S_{i}$ in $D S$.

The heights of the ordinates $B Q_{1}, C R_{1}, D S_{1}$ represent the first linear interpolates $u^{(1)}$ of $\S 2$.

II. Join $Q_{1} R_{1}$ to cut $X T$, and determine thereby a point $R_{2}$ in $C R$, just as before. Similarly join $Q_{1} S_{1}$ to cut $H X$, and determine in the same way a point $S_{2}$ in $D S$.

The heights of ordinates $C R_{2}, D S_{2}$ are the next interpolates $u^{(2)}$.

III. Join $R_{2} S_{2}$ and produce it to cut $X T$ in a point $T_{3}$.

Then the ordinate $X T_{3}$ represents the cubic interpolate $u^{(3)}$ desired.

The mode of construction is general, and can be applied to find (in theory) a desired ordinate of the polynomial curve determined by $n+1$ given ordinates.

(4) The question of remainder terms arises. In the case of one variable the method of iteration, whether quadratic or linear, achieves the effect of finding the polynomial $u_{a, b, c, d}$, for example. Now it is known ( $C f$. Steffensen, Interpolation, p. 24) that the remainder in such a representation of $u_{x}$ is given by

$$
K_{a, b, c, d}=(x-a)(x-b)(x-c)(x-d) u(x, a, b, c, d),
$$

and more conveniently for many applications, by

$$
R_{a, b, c, d}=(x-a)(x-b)(x-c)(x-d) \frac{u^{i v}(\xi)}{4 !},
$$

where $\xi$ is comprised between, or possibly coincides with one of, the smallest and the largest of the numbers $a, b, c, d, x$. This test is not always easy to apply, and in the iterative method proposed in this paper the check by convergence to equality of two iterates will in almost all cases be regarded as conclusive.

In interpolation upon two variables (Steffensen, ibid. pp. 206-212) the general remainder formula can be remembered as a symbolic product of two formulae like (8.2) above, written shortly as, e.g., $x_{(4)} D_{\xi}^{4} / 4$ ! for the case of $x$, and $y_{(6)} D_{\eta^{\prime}}^{6} / 6$ ! for $y$, say. Such a remainder term (for a cubic-quintic) would be

$$
R_{x, y}=x_{(4)} D_{\xi}^{4} / 4 !+y_{(6)} D_{\eta}^{6} / 6 !+x_{(4)} y_{(6)} D_{\xi}^{4} D_{\eta}^{6} /(4 ! 6 !)
$$

where the multiplication in the third term is of operators, and the values of $\xi$ and $\eta$ are not necessarily identical with those implied in the first two terms of the formula. From this general formula the limits to the error committed in using any particular set of values of $x$ and $y$ in the lattice of data can be theoretically ascertained. 
(5) Finally, it remains question for investigation whether the method of quadratic extrapolation may not be applied with advantage to such interpolation on a large scale as is required by subtabulation. It is obvious, for example, that subtabulation to a fifth or a tenth of the interval, as far as fifth differences, can be achieved by extrapolation with appropriate values of $\theta$ from three linear sequences of subtabulates, but the question of the time of copying down and checking remains. We reserve these considerations for more detailed examination.

\section{§9. Supplementary Remarks. [Added 12th February 1932.]}

Further consideration of $\S 4$ of the preceding paper shows that the case of interpolation for $u_{x}$ upon an odd number of symmetrically placed data $u_{0}, u_{a}, u_{-a}, u_{b}, u_{-b}, \ldots$ can be reduced to the simpler even case of $\S 3$ in various ways, which may be thought better than those already proposed. One of these ways is to compute the linear interpolates $u_{0, a}, u_{0,-a}, u_{0, b}, u_{0,-b}, \ldots$, let us say, $u_{a}^{(1)}, u_{-a}^{(1)}, u_{b}^{(1)}, u_{-b}^{(1)}, \ldots$, and to treat these as an even number of symmetrical data from which $u_{x}^{(1)}$ is to be determined just as in $\S 3$, except that in the equidistant case the quadratic multipliers will be the $\theta, 3+\theta, 8+\theta, \ldots$ of $\S 4$. Another way applicable only in the case of equidistant data $u_{0}, u_{1}, u_{2}, u_{3}, \ldots$, is to compute linear interpolates $u_{0,1}, u_{0,2}, u_{0,3}, \ldots$, that is, the $u_{1}^{(1)}, u_{2}^{(1)}, u_{3}^{(1)}, \ldots$ of $\S 2$, and to interpolate upon these for $u_{x}^{(1)}$ in the manner of $\S 3$, the multipliers being now the rather more advantageous $\theta, 2+\theta, 6+\theta, \ldots$ of that section. Yet another way is peculiarly useful when first derivatives of the tabular values of the function $u_{x}$ are provided, as may sometimes be the case: for example the normal frequency function is the derivative of the error function or probability integral, and both functions have been tabulated. In this case we can assign a meaning to $u_{0,0}$, which is readily seen to be $u_{0}+x u_{0}^{\prime}$, and this value can be computed and taken as leader of the Jordan interpolates $u_{ \pm a}, u_{ \pm b}, \ldots$, the quadratic multipliers being $-x^{2}, a^{2}-x^{2}, b^{2}-x^{2}, \ldots$ in the general case, and $\theta-1, \theta, 3+\theta, 8+\theta, \ldots$ in the equidistant case corresponding to $\S 4$.

It is not proposed to go into detail over these variants, which can easily be justified, or to give numerical examples, since no special novelty of procedure is involved; but trial has proved that all of them, and especially the last, are convenient and rapid. 
A final word may be hazarded on the question of tabulation. At present it is customary, at least in many cases, to print in addition to the functional values the central differences of even order, often as far as sixth differences, and to advocate the use of Everett's formula of interpolation, the coefficients of which have been computed and tabulated for values of $x$ from 0 to 1 at intervals of 0.001 . If interpolation is being carried out by arithmometer-and a machine is almost a necessity if a considerable number of digits is retained, whatever the process employed - then it would seem that the methods described in this paper provide a simple, expeditious and easily controlled algorithm for interpolation, which dispenses with differences. Since the choice of a smaller interval in $x$ almost always increases the convergence of formulae of interpolation in a decided fashion, the space devoted to the printing of differences might with advantage, in our opinion, be devoted to printing the tabular values for smaller intervals. This is especially the case in 2-variable interpolation, where, if differences were omitted and if the data were given at the proportionately closer intervals which this would allow, it might be possible to base accurate interpolation on no more than 16 , and possibly on 12 or on 9 central values. It would also be of use to have some indication, in default of differences, of what order of polynomial interpolation-whether cubic, quartic, and so on, and doubtless varying in different parts of a table-would ensure accuracy to any desired number of places; not necessarily the full number tabulated, since this is almost always in excess of practical requirements. Information of this kind might conveniently be inserted in tables. At the same time it is true that the routine we have described largely obviates the need for such a use of error terms, since the successive closing up of the approximation is made evident in the course of the calculations. 